\title{
Il Trittico siciliano di Brancati ${ }^{1}$
}

\author{
Cristina Coriasso Martín-Posadillo \\ Universidad Complutense de Madrid \\ cristina.coriasso@gmail.com
}

\begin{abstract}
Questa nota prende spunto dall'edizione spagnola di tre romanzi di Vitaliano Brancati (Tríptico siciliano: Don Giovanni en Sicilia, El bello Antonio, Los placeres de Paolo, Traducción de Roberto Falcó Miramontes, Ángel Sánchez-Gijón y Rosa Marcela Pericás, Lumen, Barcelona, 2009), per porre in evidenza il filo conduttore che unisce i tre romanzi che esprimono i vari aspetti e fasi dell'erotismo maschile e le influenze leopardiane che, soprattutto nell'ultimo e interrotto romanzo Paolo il caldo, si possono rilevare.
\end{abstract}

Parole chiavi: Brancati; Leopardi; Sicilia; erotismo maschile.

\section{Abstract}

This note considers the Spanish edition of three novels by Vitaliano Brancati (Tríptico siciliano: Don Giovanni en Sicilia, El bello Antonio, Los placeres de Paolo, Traducción de Roberto Falcó Miramontes, Ángel Sánchez-Gijón y Rosa Marcela Pericás, Lumen, Barcelona, 2009), to highlight the common thread that connect the three novels, that express the various aspects and phases of male eroticism, and the Leopardi influences in Brancati, especially in the last novel Paolo il caldo.

Keywords: Brancati; Leopardi; Sicily; male eroticism.

1. Questa nota prende spunto dall'edizione spagnola di Vitaliano BRANCATI, Tríptico siciliano: Don Giovanni en Sicilia, El bello Antonio, Los placeres de Paolo, traducción de Roberto Falco Miramontes, Ángel Sánchez-Gijon y Rosa Marcela Pericas, Barcelona: Lumen, 2009. 
Quasi esaurita la prima edizione (2009) spagnola dei tre principali romanzi di Vitaliano Brancati, è stata di nuovo editata da Debolsillo (2010). In entrambi i volumi si raccolgono le traduzioni pubblicate negli anni settanta e ottanta di Ángel Sanchez-Gijón (Don Juan en Sicilia, Barcelona: Bruguera, 1985), di Rosa Marcela Pericás (El guapo Antonio, Barcelona: Planeta, 1973) e quella di Roberto Falcó Miramontes, che traduce il romanzo incompiuto Paolo il caldo (Brancati muore improvvisamente in un'operazione chirurgica nel 1954) col titolo Los placeres de Paolo (2009). Un titolo quest'ultimo che, anche se attraente, non rende esplicito il filo conduttore del romanzo: vale a dire la dialettica caldofreddo, paragonabile ad altre contrapposizioni elaborate da Brancati, come nord-sud, luce-tenebra, sensualità e lussuria vs ragione, topici, questi ultimi, che accompagnano anche le avventure dell'animo dei protagonisti di Don Giovanni in Sicilia (1941) e di Il bell'Antonio (1949). Probabilmente, la citazione di un brano scritto dalla sposa, è un modo fin troppo soggettivo di presentare la figura di questo romanziere, saggista e sceneggiatore imprescindibile del XX⿳亠丷厂 secolo italiano. Trattandosi però di Anna Proclemer, ${ }^{2}$ grande e intelligente attrice, ci sembra questo uno spunto prezioso per offrirci un ritratto, seppure embrionale, della personalità intellettuale e umana del marito. L'attrice ricorda cosí la sua reazione di fronte al suo diniego d'amore:

Dopo la sua bellissima dichiarazione d'amore e la mia squallida risposta B. ritornò in Sicilia, al suo oscuro lavoro di professore magistrale. Preferiva la povertà e il silenzio della provincia alla brillante vita romana ambiguamente compromessa col regime che lui esecrava. Forse era anche un modo di autopunirsi per una sua brevissima, giovanile, dannunziana adesione al «vitalismo» fascista che aveva subito rinnegato, ma che sentiva in sé come una macchia da cancellare. E poi aveva bisogno della Sicilia. Per tutta la vita vi tornò, periodicamente, come a un grembo segreto, madre, linfa, ispirazione. ${ }^{3}$

Due elementi essenziali si possono ricavare da queste parole; da un lato: che cos'è tutta l'arte di Brancati, incluso il teatro e il cinema, nonché i romanzi, se non l'impegno di cancellare quanto di ideologico ed enfatico c'era nella sua fase iniziale di scrittore alle prime armi, servendosi del comico e del grottesco, cercando però di ottenere, come in un complicato processo alchimico, quelle rare gocce di vero sublime che tanto abbondano nella sua produzione e che facevano dire a Sciascia, suo allievo, che quello e non altro era il modo in cui voleva scrivere? E dall'altra parte, secondo elemento fondamentale, la Sicilia, madre, grembo, ispirazione, in altre parole, mito vissuto nella lucida coscienza di ogni mistificazione, luce del sud contenente le tenebre, come bene esprime il personaggio di Paolo Castorini nel piú biográfico, ultimo e incompiuto romanzo di Brancati, Paolo il Caldo: «Lo sforzo constante della mia vita è stato di vedere la luce del mondo (che per me è quella della Sicilia) dalla parte

2. Ha pubblicato e commentato il rapporto epistolare: Vitaliano BranCATI, Anna Proclemer, Lettere da un matrimonio, Milano: Rizzoli, 1978.

3. Ibid., p. 10. 
ridente, ed espellere dal cervello le influenze della sua ripresa buia, dalla quale derivano l'apprensione e la lussuria». ${ }^{4}$ Infatti, nel descrivere la dialettica lucetenebre che caratterizza il cielo del meridione, con i suoi sensi allegorici, la capacità di Brancati di unire un'amorosa evocazione ad una lucida demitificazione non ha forse paragone nel nostro tempo. Da una parte "[...] il silenzio meridiano è assordante come un tuono», 5 dall'altra:

[...] nonostante la sua intensità, o forse a causa di questa, la luce del sud rivela nella memoria una profonda natura di tenebra. Nella sua esorbitanza, varca continuamente i confini del regno opposto, e quando si dice che è accecante, si vuole forse alludere, senza averne esatta coscienza, a certi guizzi di buio che vengono dal suo interno, a certi squarci sulla notte cupa come può farli un'eclissi nel cielo di mezzogiorno, salvo che questi sono lenti e progressivi e, una volta chiusi, non riaprono più, e quelli invece rapidi e continui, sicché la sensazione della luce per chi, insospettito della propria malinconia o tetraggine, voglia esaminarla, risulta composta di due sensazioni contrarie, di chiaro e di oscuro, alternate fulmineamente, in modo che l'impressione totale è di chiaro. ${ }^{6}$

Nella traduzione di Roberto Falcò Miramontes ${ }^{7}$ —elegante e corretta-, si traduce accecante per deslumbrante, invece di usare la parola cegadora - che sarebbe il correlato esatto- una saggia scelta, a mio avviso, che rende meglio la paradossale trasformazione dei contrari dove la luce piú potente diventa tenebra e, nel piano del sentimento, la piú furiosa vita, l'anticamera di una cupa tetraggine. Ma questo paradossale esito dell'eccesso di vita e sensualità del carattere del sud, trasformato in lucida e rassegnata distanza, tinta di comico, non è che lo sviluppo che fa Brancati dell'analisi di Leopardi, del quale aveva elaborato una antologia di testi riguardanti la lingua e la letteratura d'Italia, ${ }^{8}$ e che tanto nello Zibaldone come nel Saggio sopra lo stato presente dei costumi degli italiani (1824) esprime la sua teoría sull'estremo caldo del sud, físico e morale, che nell'eccesso si trasforma nel suo perfetto contrario:

4. Vitaliano Brancati, Paolo il caldo, Milano: Mondadori, 2010, p. 15.

5. Ibid., p. 11.

6. Ibid., p. 12.

7. «Y, sin embargo, a pesar de su intensidad, o quizá a causa de ella, la luz del sur revela en la memoria una profunda naturaleza de tiniebla. En su exorbitancia, rebasa continuamente los confines del reino opuesto, y cuando se dice que es deslumbrante, se quiere aludir tal vez, sin tener conciencia exacta, a ciertos destellos de oscuridad procedentes de su interior, a ciertas fisuras en la noche oscura como las que puede abrir un eclipse en el cielo de mediodía, salvo que estas son lentas y progresivas y, una vez cerradas, no se reabren más, mientras que aquellas, por el contrario, son rápidas y continuas, de modo que la sensación que causa la luz en quien, escamado por la propia melancolía o tristeza, quiera examinarla resulta una mezcla de dos sensaciones contrarias, el claro y el oscuro, alternadas de manera vertiginosa, de modo que la impresión general es de claridad». BranCatı, Tríptico siciliano: Don Giovanni en Sicilia, op. cit., p. 498.

8. Giacomo Leopard, Lingua, letteratura e società in Italia, a cura di Vitaliano Brancati, Milano: Bompiani, 1942. 
È tutto mirabile e simile a paradosso, quanto vero, che non v'ha né individuo né popolo sì vicino alla freddezza, all'indifferenza, all'insensibilità e a un grado così alto e profondo e costante di freddezza, insensibilità e indifferenza, come quelli che per natura sono più vivaci, più sensibili, più caldi. Collocati questi tali o popoli o individui in uno stato e in circostanze o politiche o qualunque, in cui niuna cosa conferisca all'immaginazione e all'illusione, anzi tutto contribuisca al disinganno, questo disinganno per la vivacità stessa della loro natura $\mathrm{e}$ in ragione diretta di essa vivacità è completo, totale, fortissimo, profondissimo. L'indifferenza che ne risulta è perfetta, radicatissima, costantissima; l'inattività, se si può così dire, efficacissima; la noncuranza effettivissima; la freddezza è vero ghiaccio, come accade nel gran caldo che i vapori sono da esso elevati a tanta altezza che quivi stringendosi nel più duro gelo, precipitano ridotti in gragnuola. I popoli settentrionali meno caldi nelle illusioni, sono anche meno freddi nel disinganno. ${ }^{?}$

Quest'opera di Leopardi era senza dubbio presente nella mente di Brancati quando scriveva: «Italia, italiani, usi e costumi degli italiani: ecco una materia facile per anni di malumore. Sulla volgarità e la rozzezza, chi di noi può scrivere una frase elegante?» ${ }^{10}$

Paolo il caldo è senza dubbio il più impegnativo dei tre romanzi, quello in cui la riflessione e la meditazione sostengono il tessuto delle scene che si susseguono, e dove il personaggio di Michele, padre di Paolo, è il contrappunto morboso e razionale della sensualità quasi bestiale del nonno Paolo e dello zio e, che portata agli estremi, è vissuta alla fine come lussuria, ossessione torbida che assorbe e annulla ogni forza creativa del protagonista. Sembra che nell'anima del protagonista quella figura, esplicitamente leopardiana, ${ }^{11}$ del padre diviso fra i libri e i suoi mali fisici e mentali, lotti inutilmente per vincere un'altra forza, rappresentata da tutta la società siciliana nel neurotico connubio di Chiesa e Fascio, esaltazione della donna ed erotismo che denigra la donna, che lo attira inesorabilmente verso il basso. Un dissidio che non si

9. Giacomo Leopardi, Discorso sopra lo stato presente dei costumi degli italiani, edizione di note e introduzione di Mario Andrea Rigoni, testo critico di Marco Dondero, commento di Roberto Melchiori, Milano: Rizzoli, 1998, p. 79.

10. Brancati, Paolo il caldo, op. cit., p. 9.

11. Ecco la descrizione del personaggio del padre, Michele, malato di cuore a causa, secondo viene detto, di una malattia di contagio sessuale trasmessa dal nonno Paolo: «Quando il verbo era il soggetto si riferiva sempre al cuore. "Non avrei tempo, fra un battito e l'ltro, di pensare che s'è fermato per sempre. Non credere però che abbia paura della morte. Mi danno soltanto un gran fastidio questi lunghi minuti di silenzio, dentro il petto... Leopardi, lo so" aggiunse mentre le sue dita, percorrendo uno scaffale, capitavano sul dorso delle Operette morali, "era pallido come me, soffriva piú di me. Ma ci sono sofferenze che scavano nella persona come i buchi di un flauto, e la luce dello spirito ne esce melodiosa, altre invece, come la mie, assorbono tutta l'attenzione e incantano l'intelligenza... Un uomo rimane chiuso nel cerchio del suo corpo, e non produce che sbadigli e silenzio... Però", aggiunse ancora con la voce energica, voltandosi verso Paolo, "torno a ripetere chi io solo fra di voi ho un'idea chiara della felicità. Il mio mal di testa è meno sordo della stanchezza che vi prende, te, tuo zio e tuo nonno, dopo che avete fatto certe cose".» Ibid., p. 74. 
risolve e che si polarizza man mano si procede nella lettura dei tre romanzi che, come indica il titolo dell'edizione spagnola, formano un Tríptico, una triade che illustra i tre esiti di questa sofferenza propria dell'erotismo maschile. In Giovanni Percolla il dissidio resta nell'immaginazione senza distruggere l'esito borghese e felice: il gallismo, più immaginato che vissuto, sopravvive al controllo della sposa-madre. Invece il protagonista di Il bell'Antonio è minimamente incapace di unire il desiderio sessuale all'immagine d'angelo del suo ideale. Tutto ciò è come una sorta di evoluzione dell'amore cortese, stilizzato ed esemplificato dai versi leopardiani (appartenenti al Canto XVII, Consalvo, vv. 125-127), trascritti da Brancati nella dedica alla sposa all'inizio del romanzo: («...Ahí, lice in terra / Provar felicità. Ciò seppi il giorno / che fiso io ti mirai...»). La pressione psicologica e sociale che Antonio sopporta sbocca tragicamente nell'impotenza e nello scandalo. L'esito di Paolo Castorini rappresenta l'aspetto contrario, non è una mistificazione dell'amore a rendere sterile il desiderio, ma un desiderio incapace di manifestarsi in un contatto con la donna amata, che porta il protagonista a ricadere in una lussuria che senza soddisfarlo lo allontana dall'amore e dalla creatività: nonostante sia lei ad andarsene, è lui che non ha saputo adeguare la sua sessualità ad una relazione al di là della divaricazione fra la donna come santa e la donna come puttana. Un cammino, quello delle fasi erotiche dell'uomo, che Brancati percorre, investiga e chiarisce, e che resta lontano dalla banalità erotica e dall'esaltazione del grottesco costume, perche arriva fino alle radici del desiderio inerente all'essere, cercando nella letteratura, come già nella vita, una via di scampo. Troveranno quindi i lettori di questa raccolta di Brancati in spagnolo, molto di più che un ritratto gioioso del pittoresco sud; troveranno, nei casi in cui la traduzione ha saputo ricrearne lo stile, tutta la magia del Brancati che sa far ridere a proposito della mistificazione di un contenuto mitico e che allo stesso tempo fa gioire della più classica e radiante Bellezza. Ecco un esempio di questo equilibrio:

La será era già inoltrata. Spense la lampada e gettò la camera nel buio, con l'effetto di accendere sui vetri l'immagine della grande scogliera di tetti e terrazze, bagnata dal cielo inazzurrato da un invisibile quarto di luna e serpeggiata da luci rosse di fanali, fosforescenze di vetture-pesci che roteavano in tutti i sensi, una di queste filando però diritta verso di lui. In cima a Monte Mario, nel cielo alto, due fari versavano di quando in quando una goccia di sangue; un filobus, stridendo con l'asta di presa contro il filo, mandò un lampo viola che mise in risalto il grande spazio vuoto che aveva per base tutta la larghezza del Tevere, del quale piano piano, nel silenzio, si andava percependo il fruscio di seta, rumore delicatamente femminile che rendeva oltremodo struggente quell'immagine di città al di lá dei vetri, viva e candidissima ora, per un aumento della luce lunare, come la stessa dea Venere scesa nel balcone ad annunziare, con la sua nudità celeste, l'avvicinarsi di una delle sue infinite copie mortali in cui ella ha però lasciato cadere, nell'atto di tentarla e persuaderla, una goccia del suo turbato sesso divino. La città, generatrice inesauribile di sconosciute; strade, piazze, edifici, per i quali egli 
sentiva minutamente muoversi la carne della donna, questo elemento infido, minaccioso ed attirante, il solo in cui l'universo smetta di essere duro e freddo come la pietra di una tomba. ${ }^{12}$

E la riuscita versione di Falcò:

Empezaba a caer la tarde. Apagó la lámpara y sumió la habitación en la oscuridad, lo que creó el efecto de que se había encendido en los cristales la imagen del gran arrecife de tejados y terrazas, bañado desde el cielo teñido de azul por un invisible cuarto de luna y salpicado de las luces rojas de las farolas, con los destellos fosforescentes de los coches-peces que circulaban en todos los sentidos, uno de los cuales se dirigía directo hacia él. En la cima del monte Mario, en el cielo, dos faros derramaban de vez en cuando una gota de sangre; un trolebús, que avanzaba bajo los chispazos causados por la fricción del trole en el cable eléctrico, provocó un relámpago violeta que resaltó el gran espacio vacío que se extendía a lo largo del Tíber, en el que poco a poco en el silencio se percibía el crujido de la seda, el ruido delicadamente femenino que convertía la imagen de la ciudad que se veía tras los cristales en una sensación tremendamente desgarradora, una imagen viva y candidísima, debido al aumento de la luz de la luna, como si la propia diosa Venus hubiera bajado al balcón para anunciar, con su celeste desnudez, la llegada de una de sus infinitas copias mortales, en la que sin embargo, había dejado caer, en el acto de tentarla y persuadirla, una gota de su turbado sexo divino. La ciudad, generadora inagotable de desconocidas; calles, plazas, edificios por los que Paolo sentía minuciosamente moverse la carne de la mujer, este elemento peligroso, amenazador y atrayente, el único en el que el universo deja de ser duro y frío como la lápida de una tumba. ${ }^{13}$

Se è possibile, considero adeguato non accorciare o troncare il periodo e conservare la complessità sintattica dell'originale che lo spagnolo può tollerare senza «italianizzarsi». In generale la dialettica fedeltà-libertà è stata ben colta nella traduzione di questo bellissimo brano; è opportuno il cambiamento di "pietra» per lápida invece di piedra: in effetti, "pietra» nel tono alto dell'originale ha connotazioni sepolcrali e barocche che permettono senza dubbio tale traduzione, giacché, d'altra parte, il termine piedra in spagnolo, a mio parere, no si usa in questa accezione. Il traduttore ha saputo trasmettere l'essenziale: non giá il semplice contenuto ma il tono, per un verso comico e demistificatorio, per un altro verso, cosmico e sublime, senza abbandonare la lucidità e l'ironia: la carne della donna, sentita come attrazione e repulsione secondo la sintomatica del sublime, quasi a rappresentare l'eros come l'unica sfida possibile alla materia inerte nella quale consiste l'essere delle cose.

12. Ibid., p. 198-199.

13. Brancati, Tríptico siciliano..., op. cit, p. 737-738. 